SOI: 1.1 /TAS DOI: $10.15863 /$ TAS

International Scientific Journal Theoretical \& Applied Science

p-ISSN: 2308-4944 (print) e-ISSN: 2409-0085 (online)

Year: $2015 \quad$ Issue: $11 \quad$ Volume: 31

Published: $30.11 .2015 \quad \underline{\text { http://T-Science.org }}$
Vjacheslav Vadimovich Syskov Joint Stock Company «Central research institute of economy, informatics and control systems»»

Ph. D. Tech. Sc., Chief Researcher, Department of Modeling and Control Systems slavacestmoi@gmail.com

Vadim Vladimirovich Borisov Dr. Tech. Sc., Professor Branch of the National Research University «MPEI» in Smolensk vadim.v.borisov@mail.ru

SECTION 4. Computer science, computer engineering and automation.

\title{
METHOD OF CONSISTENT DESCRIPTION OF COLLECTIVE ACTIVITY IN COMPLEX ORGANIZATIONAL AND TECHNICAL SYSTEMS
}

\begin{abstract}
The problem of the accounting of essential features of collective activity at formalization and development of activity models in complex organizational and technical systems is designated. The method of consistent description of collective activity directed at keeping the peculiar properties of collective activity, such as the coordinated performance of work, the accounting of results of joint activity and operating mode of divisions, subjectivity of officials behavior and uncertainty of clients behavior, control of tasks with an independent order is executed. The reported study was partially supported by RFBR, research project No. 14-07-00338_a, and by Ministry of Education of Russia, project No. 2493 in basic part of the state task No. 2014/123.

Key words: business process, collective work, description of activity, activity model, model of collective activity, business process modeling, business process management.

Language: Russian

Citation: Syskov VV, Borisov VV (2015) METHOD OF CONSISTENT DESCRIPTION OF COLLECTIVE ACTIVITY IN COMPLEX ORGANIZATIONAL AND TECHNICAL SYSTEMS. ISJ Theoretical \& Applied Science 11 (31): 34-52.

Soi: http://s-o-i.org/1.1/TAS-11-31-8 Doi: crossef http://dx.doi.org/10.15863/TAS.2015.11.31.8

\section{МЕТОД СОГЛАСОВАННОГО ПРЕДСТАВЛЕНИЯ КОЛЛЕКТИВНОЙ ДЕЯТЕЛЬНОСТИ В СЛОЖНЫХ ОРГАНИЗАЦИОННО-ТЕХНИЧЕСКИХ СИСТЕМАХ}

Аннотация: В статье обозначена проблема учета особенностей коллективной деятельности при формализации и разработке моделей деятельности в сложных организационно-технических системах. Для ее решения предложен метод описания деятельности, позволяющий учитывать существенные особенности коллективной деятельности, такие как согласованное выполнение работ, учет результатов совместной деятельности и режсиов работы подразделений, индивидуальности поведения должностных лиц и неопределенности поведения клиентов, контроль нерегламентированных определенным порядком заданий.

Ключевые слова: бизнес-прочесс, коллективная деятельность, описание деятельности, модель деятельности, модель коллективной деятельности, моделирование бизнес-проиессов, процессное управление.
\end{abstract}

Со времени появления концепции Business Process Management (ВРМ) основной из форм реализации процессного управления до сих пор является формализованное описание и графическое моделирование деятельности, часто называемое «чистым документированием и регламентацией бизнес-процессов».

Применение этой формы обосновывается необходимостью задания порядка выполнения процессов при анализе, упорядочении и обосновании возможности реорганизации деятельности.

При этом использование современных систем процессного управления для документирования и регламентации не всегда позволяет полностью решить проблемы, связанные с представлением коллективной деятельности в организационно-технических, производственных или социально-экономических системах. В этом случае эти проблемы возникают из-за трудности выявления (при работе бизнес- 
аналитика) или невозможности отражения (самой ВРМ-системой) различных особенностей коллективной деятельности с помощью существующих средств реализации процессного управления. К этим особенностям относится:

- согласованное выполнение работ;

- учет режимов и графиков работы подразделений, индивидуальности поведения должностных лиц и неопределенности поведения клиентов;

- контроля заданий, нерегламентированных определенным порядком выполнения; работы.

- использования результатов совместной

Целью данной статьи является обоснование метода согласованного представления для реализации эффективной коллективной деятельности в сложных организационнотехнических системах в части ее формализации и моделирования.

Назначение метода согласованного представления коллективной деятельности

\begin{tabular}{lcrr}
\multicolumn{1}{c}{ Метод } & согласованного & \multicolumn{2}{r}{ представления } \\
коллективной & \multicolumn{2}{c}{ деятельности } & должен \\
устанавливать & систематизированный & порядок \\
действий & описывать & новые & или \\
усовершенствованные способы & (приемы) & для \\
учета существенных особенностей коллективной \\
деятельности в разрабатываемых с его помощью \\
моделях деятельности.
\end{tabular}

Этот метод должен позволять проводить формализацию и графическую разработку моделей деятельности, отражающих разнохарактерность ее выполнения (согласованность, субъективность, последовательность или отсутствие жесткого порядка), а также связь с различными источниками данных, бизнес-правилами и другими условиями выполнения работ (отдельных единиц деятельности - от действия или задания до процесса).

При этом метод должен обеспечивать:

- адекватность представления относительно реальных процессов;

- подробность формализации в соответствии с назначением.

Необходимая степень подробности представления определяет уровень описания от концептуальных моделей до очень точных и детальных моделей деятельности, учитывающих все нюансы поведения.
Более того, модели деятельности, разработанные на основе метода согласованного представления коллективной деятельности, должны быть применимы для расширения возможностей процессного управления за счет их использования при организации деятельности, оперативном управлении и поддержке принятия управленческих решений в сложных организационно-технических системах. Целесообразность последующего применения моделей обосновывается тем, что полное формализованное описание деятельности «лучшее “руководство" для настройки исполнения целевых бизнес-процессов, а также составляющих их конкретных процедур средствами выбранного прикладного программного обеспечения» [1].

\section{I. Описание метода согласованного} представления коллективной деятельности

Метод согласованного представления коллективной деятельности, согласно рис. 1 , включает этапы:

- анализа коллективной деятельности;

- выполнения описания коллективной деятельности.

В ходе анализа коллективной деятельности проводится:

Выявление процессов;

Установление факта, что деятельность является единым процессом;

Декомпозиция процессов;

Уточнение перечня процессов.

Далее, при выполнении описания осуществляется:

Построение процессной архитектуры;

Описание сложных процессов;

Описание простых процессов.

Помимо порядка выполнения этапов и шагов, данная схема определяет использование признаков процессов, типовых вариантов взаимодействия работ в рамках процесса, а также способов представления, которые должны обеспечивать:

- формализованное описание коллективной деятельности;

- графическое моделирование коллективной деятельности. 


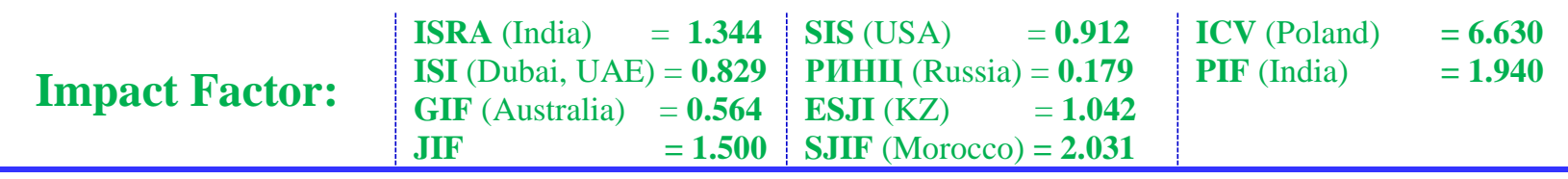

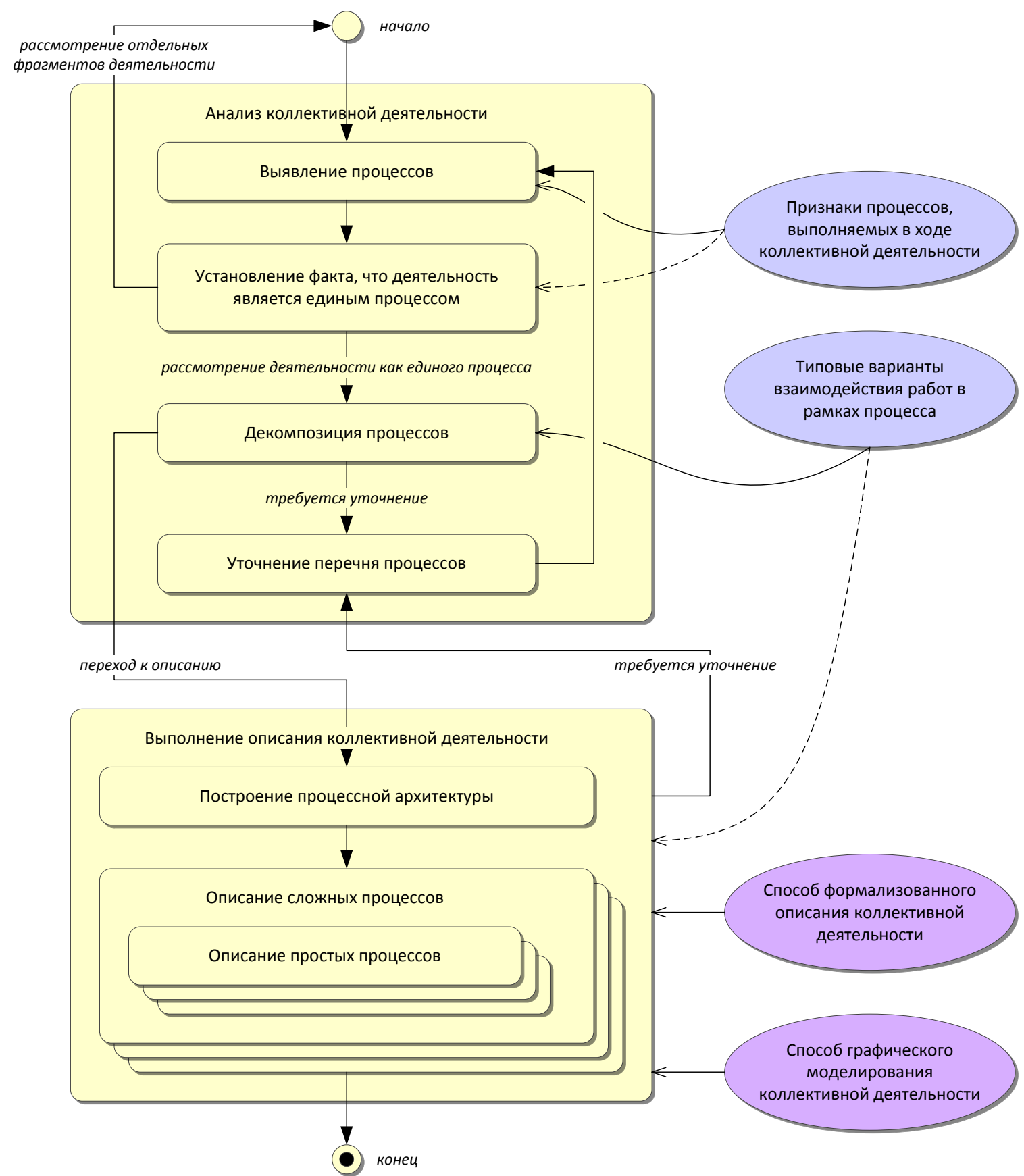

Рисунок 1 - Схема метода согласованного представления коллективной деятельности

\section{Этап 1. Анализ коллективной деятельности}

На первом этапе метода выделяются и идентифицируются отдельные крупные составляющие коллективной деятельности процессы.

\section{Шаг 1. Выявление процессов}

При выявлении процессов составляется перечень всех процессов и некоторых (явно указанных для них) артефактов, таких как условие начала, продолжительность и т. п. С этой целью изучаются стандарты, нормативная, техническая и другая документация, относящаяся к работе рассматриваемой системы.

При этом для процессов проверяется наличие признаков, позволяющих их отнести к процессам, выполняемым в рамках коллективной деятельности. Основные признаки процессов, выделенные на основании анализа [2], приведены в табл. 1. Для конкретной предметной области этот перечень может быть уточнен.

ISPC Technological advances, 
Таблица 1

\section{Основные признаки процессов}

\begin{tabular}{|l|l|}
\hline \multicolumn{1}{|c|}{ Наименование } & \multicolumn{1}{|c|}{ Описание признака } \\
\hline Повторяемость & Процессом является лишь повторяемая деятельность \\
\hline Предсказуемость & $\begin{array}{l}\text { Процессом является деятельность, развивающаяся по предсказуемым заранее (на } \\
\text { этапе проектирования) сценариям выполнения отдельных заданий или действий }\end{array}$ \\
\hline Результативность & $\begin{array}{l}\text { Процессом является деятельность, ведущая к появлению некого ценного результата } \\
\text { или нацеленная на эффективность }\end{array}$ \\
\hline $\begin{array}{l}\text { Нетривиальность } \\
\text { (делимость) }\end{array}$ & $\begin{array}{l}\text { Процессом является деятельность, которая может быть декомпозирована на } \\
\text { множества связанных различным образом единиц работы }\end{array}$ \\
\hline $\begin{array}{l}\text { Направленность на } \\
\text { объект }\end{array}$ & $\begin{array}{l}\text { Процессом является деятельность, ориентированная на объект, над которым } \\
\text { выполняется работа }\end{array}$ \\
\hline
\end{tabular}

Шаг 2. Установление факта, что деятельность является единым процессом

Далее, на основании анализа перечня процессов, устанавливается (или нет) факт, что вся деятельность является единым процессом. Например, в случае, если рассматриваемая деятельность не повторяется, мы имеем дело с проектом, а не процессом.

Если рассматриваемая деятельность не является единым процессом, то рассмотрение фрагментов деятельности, выявленных на первом шаге, осуществляется по отдельности (возврат к началу на рис. 1). При этом для процессов или их групп, не связанных процессными отношениями, метод начинает выполняться сначала в виде нового экземпляра. Для приведенного примера, проект может включать несколько отдельных процессов, которые можно описать с использованием предлагаемого метода, а управлять ими с помощью инструментария проектного подхода, т. е. «координировать деятельность между владельцами бизнеспроцессов и согласовывать управление ими в рамках проекта», что также является обоснованным решением [3; 4].

Если установлено, что рассматриваемая деятельность является единым процессом, то выполняется переход к декомпозиции процессов.

\section{Шаг 3. Декомпозиция процессов}

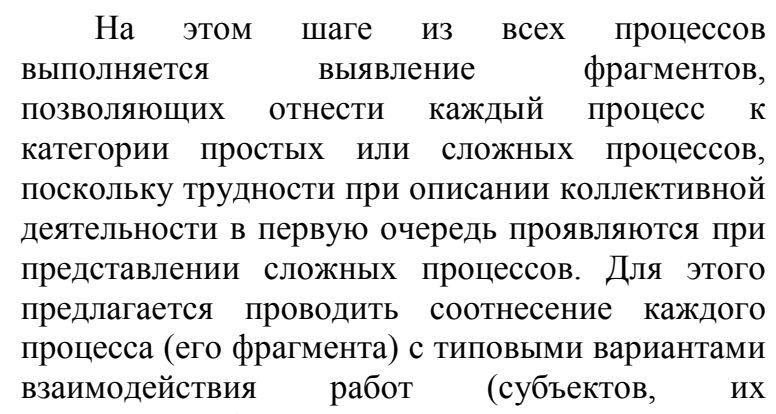
взаимодействия работ (суб
выполняющих) в рамках процесса.

Определим типовые варианты исходя из проявления основных свойств коллективной деятельности индивидуальность, (последовательность, вложенность и согласованность) [5], поскольку установление наличия этих свойств, особенно нескольких одновременно, позволяет отнести процесс к той или иной категории. Для этого в табл. 2 приведем соответствующие этим типовым варианты:

- проявления единственного (1-элементного размещения) свойства;

- парного проявления (2-элементного сочетания) свойств коллективной деятельности.

Варианты проявления свойств коллективной деятельности

\begin{tabular}{|c|c|c|}
\hline № варианта & Условное наименование варианта & Свойства \\
\hline 1 & Последовательное выполнение работ & Последовательность \\
\hline 2 & Вложенное выполнение работ & Вложенность \\
\hline $3, \mathrm{a}$ & Вложенное выполнение последовательных работ & \multirow{2}{*}{$\begin{array}{l}\text { Последовательность } \\
\text { Вложенность }\end{array}$} \\
\hline 3,6 & Последовательное выполнение вложенных работ & \\
\hline 4 & Индивидуальное выполнение работ & Индивидуальность \\
\hline 5 & Согласованное выполнение работ & Согласованность \\
\hline $6, a$ & Одновременное выполнение последовательных работ & Согласованность \\
\hline
\end{tabular}




\begin{tabular}{|c|c|c|c|c|c|c|}
\hline Impact Factor: & $\begin{array}{l}\text { ISRA (India) } \\
\text { ISI (Dubai, UAE } \\
\text { GIF (Australia) } \\
\text { JIF }\end{array}$ & $\begin{array}{l}=1.344 \\
=0.829 \\
=0.564 \\
=1.500\end{array}$ & $\begin{array}{l}\text { SIS (USA) = } \\
\text { PИНЦ (Russia) } \\
\text { ESJI (KZ) } \\
\text { SJIF (Morocco) }\end{array}$ & $\begin{array}{l}=0.912 \\
=0.179 \\
=1.042 \\
=2.031\end{array}$ & $\begin{array}{l}\text { ICV (Poland) } \\
\text { PIF (India) }\end{array}$ & $\begin{array}{l}=6.630 \\
=1.940\end{array}$ \\
\hline
\end{tabular}

\begin{tabular}{|c|l|l|}
\hline 6, б & Последовательное выполнение одновременных работ & Последовательность \\
\hline 7 & $\begin{array}{l}\text { Последовательное выполнение индивидуальных работ } \\
\text { (индивидуальное выполнение последовательных работ) }\end{array}$ & $\begin{array}{l}\text { Последовательность } \\
\text { Индивидуальность }\end{array}$ \\
\hline 8 & $\begin{array}{l}\text { Одновременное выполнение вложенных работ (вложенное } \\
\text { выполнение одновременных работ) }\end{array}$ & $\begin{array}{l}\text { Согласованность } \\
\text { Вложенность }\end{array}$ \\
\hline 9 & $\begin{array}{l}\text { Индивидуальное выполнение вложенных работ (вложенное } \\
\text { выполнение индивидуальных работ) }\end{array}$ & $\begin{array}{l}\text { Вложенность } \\
\text { Индивидуальность }\end{array}$ \\
\hline 10 & $\begin{array}{l}\text { Одновременное выполнение индивидуальных работ } \\
\text { индивидуальное выполнение одновременных работ) }\end{array}$ & $\begin{array}{l}\text { Согласованность } \\
\text { Индивидуальность }\end{array}$ \\
\hline
\end{tabular}

Рассмотрение 2-элементных сочетаний для выявления сложных процессов будет достаточным, поскольку сочетания из 3-х или 4-х элементов из 4-х в виду природы процесса всегда можно представить в виде нескольких сочетаний из 2-х элементов.

Количество размещений определяется числом наборов по одному из 4-х различных элементов, т. е. $A_{4}^{1}=4$ (варианты $\left.1,2,4,5\right)$, а сочетаний - числом сочетаний из 4-х элементов по 2, т. е. $C_{4}^{2}=6$ (варианты 3, 6-10).

Приведем условные схемы и интерпретацию вариантов, основанных на сочетании свойств коллективной деятельности. Вариант «3, а» может быть интерпретирован как выполнение работ, состоящих из более мелких последовательных работ, или взаимодействие зависимых субъектов с установленным порядком работы согласно рис. 2.

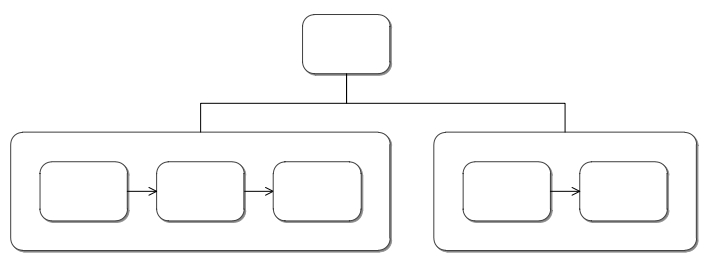

Рисунок 2 - Условная схема варианта «3, а»

Вариант «3, б» в соответствии с рис. 3 описывает поэтапное выполнение работ, состоящих из более мелких работ, или последовательное (продолжительное во времени) взаимодействие групп зависимых субъектов с установленным распределением работ.

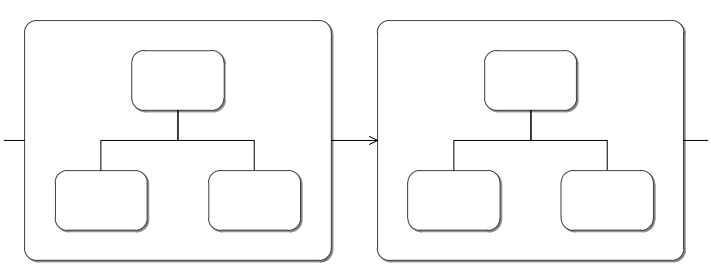

Рисунок 3 - Условная схема варианта «3, б»

Вариант «6, а» согласно рис. 4 может быть интерпретирован как последовательное выполнение согласуемых работ или регламентированное независимых субъектов, каждый из которых является сущностью («из внешнего мира» относительно другого субъекта) со своим ритмом работы.

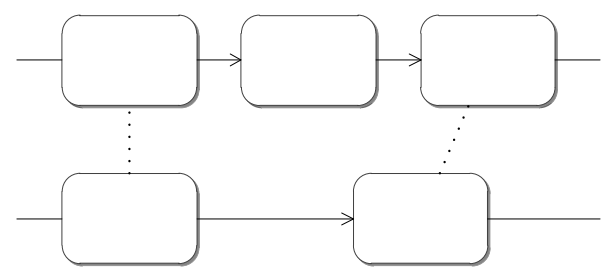

Рисунок 4 - Условная схема варианта «6, а» 


\begin{tabular}{l|lrl|l|ll} 
& ISRA (India) & $=\mathbf{1 . 3 4 4}$ & SIS (USA) & $=\mathbf{0 . 9 1 2}$ & ICV (Poland) & $\mathbf{= 6 . 6 3 0}$ \\
Impact Factor: & ISI (Dubai, UAE) $=\mathbf{0 . 8 2 9}$ & PUH (Russia) $=\mathbf{0 . 1 7 9}$ & PIF (India) & $=\mathbf{1 . 9 4 0}$ \\
& GIF (Australia) & $\mathbf{0 . 5 6 4}$ & ESJI (KZ) & $=\mathbf{1 . 0 4 2}$ & & \\
& JIF & $\mathbf{1 . 5 0 0}$ & SJIF (Morocco) $=\mathbf{2 . 0 3 1}$ & & \\
\hline
\end{tabular}

Вариант «6, б» описывает поэтапное субъектов с согласованным поведением в выполнение согласованных работ или последовательное взаимодействие групп соответствии с рис. 5 .

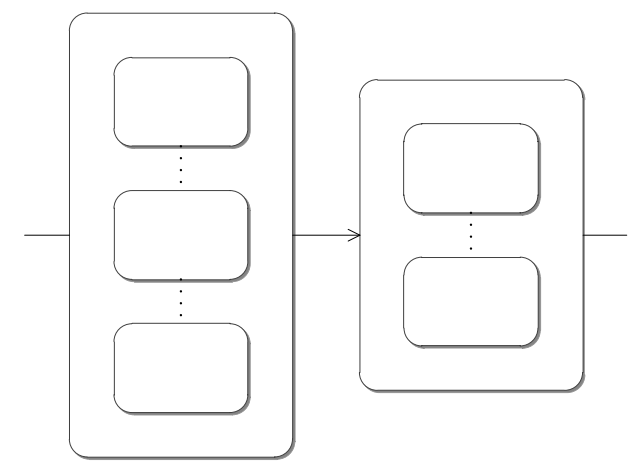

Рисунок 5 - Условная схема варианта «6, б»

Вариант «7» согласно рис. 6 предполагает поэтапное нерегламентированных определенным порядком работ или последовательное взаимодействие субъектов с индивидуальным поведением.

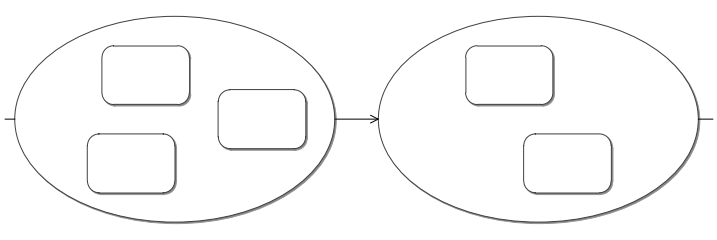

Рисунок 6 - Условная схема варианта «7»

Вариант «8» может быть интерпретирован как согласованное выполнение работ, состоящих из более мелких работ, или соподчиненное взаимодействие субъектов с установленным распределением работ в соответствии с рис. 7.

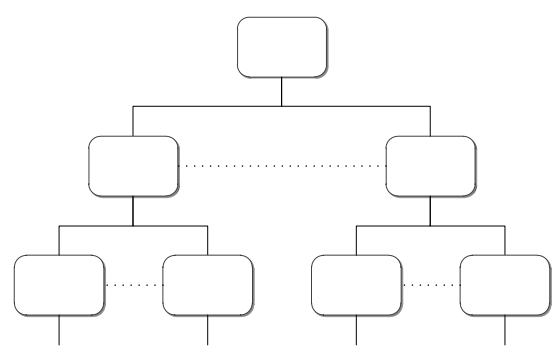

Рисунок 7 - Условная схема варианта «8»

Вариант «9» согласно рис. 8 может быть интерпретирован как выполнение нерегламентированных определенным порядком работ, состоящих из более мелких работ, или соподчиненное взаимодействие субъектов с индивидуальным поведением. 


\begin{tabular}{|c|c|c|c|c|c|c|}
\hline Impact Factor: & $\begin{array}{l}\text { ISRA (India) } \\
\text { ISI (Dubai, UAE } \\
\text { GIF (Australia) } \\
\text { JIF }\end{array}$ & $\begin{array}{l}=1.344 \\
=0.829 \\
=0.356 \\
=1.500\end{array}$ & $\begin{array}{l}\text { SIS (USA) } \\
\text { PИHЦ (Russia) } \\
\text { ESJI (KZ) } \\
\text { SJIF (Morocco) }\end{array}$ & $\begin{array}{l}=0.912 \\
=0.179 \\
=1.042 \\
=2.031\end{array}$ & ICV (Poland) & $=6.630$ \\
\hline
\end{tabular}

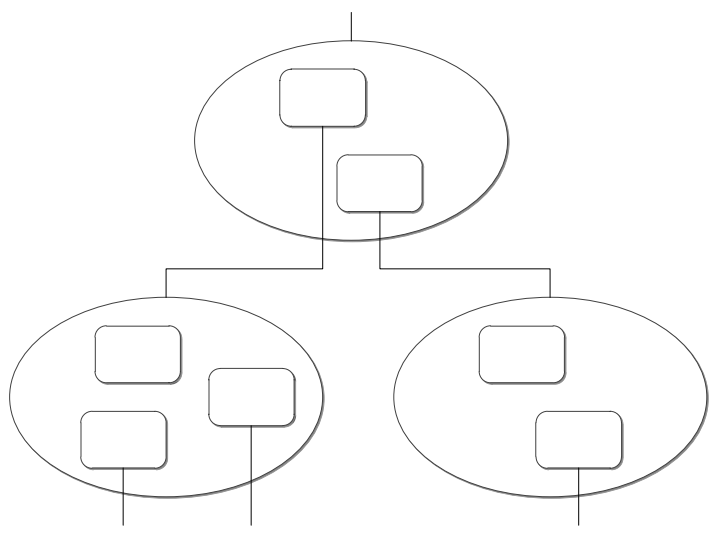

Рисунок 8 - Условная схема варианта «9»

Вариант «10» в соответствии с рис. 9 описывает согласованное выполнение групп нерегламентированных определенным порядком работ или единовременное независимых субъектов, индивидуальным поведением. взаимодействие обладающих

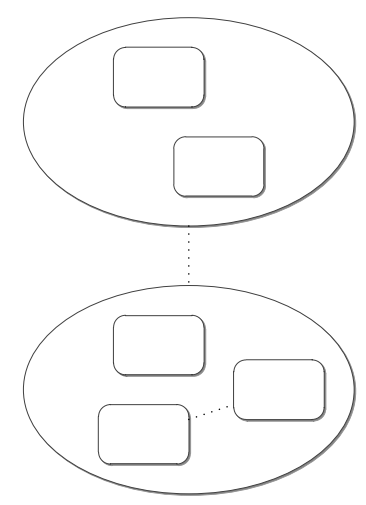

Рисунок 9 - Условная схема варианта «10»

Предполагается, что соответствие представленных вариантов позволит отнести его к определенной категории, а также применить при его дальнейшем описании соответствующий прием.

Расположение всех вариантов проявления (1-элементных размещений и парных сочетаний) свойств коллективной деятельности можно представить графически, как показано на рис. 10. Это позволяет обобщить данные о сложности типовых вариантов взаимодействия работ.
На основании анализа расположения вариантов относительно друг друга можно сделать вывод о повышении сложности при проявлении свойств индивидуальности и согласованности в сочетании с другими свойствами.

Согласно табл. 3 предлагается следующая градация сложности процессов:

- простой процесс имеет очень низкую или низкую сложность;

- сложный процесс имеет среднюю или высокую сложность. 


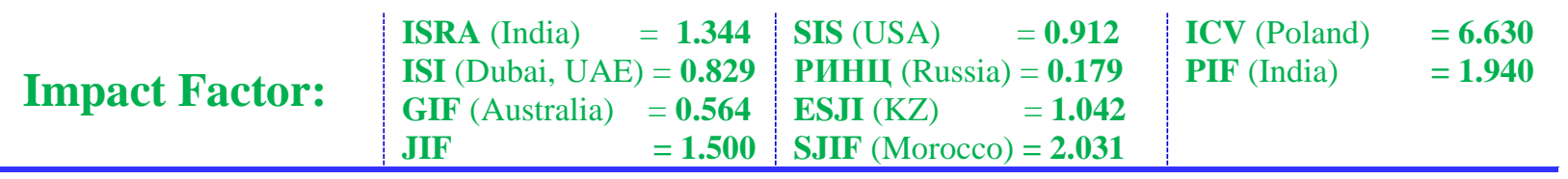

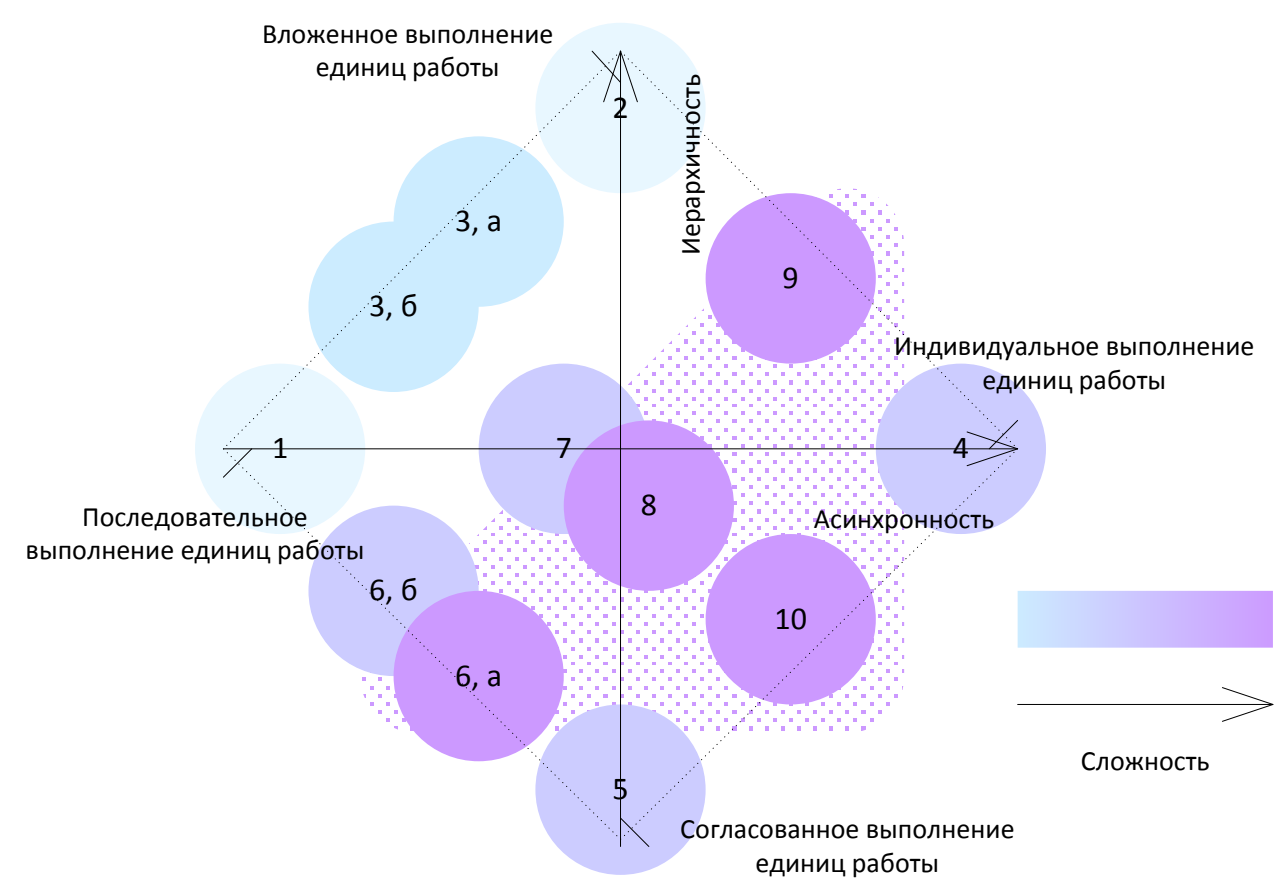

Рисунок 10 - Сложность коллективной деятельности

Таблица 3

Сложность типовых вариантов проявления свойств коллективной деятельности

\begin{tabular}{|c|l|c|}
\hline № варианта & \multicolumn{1}{|c|}{ Наименование варианта } & Сложность \\
\hline 1 & Выполнение последовательных работ & Очень низкая \\
\hline 2 & Выполнение работ, состоящих из более мелких работ & Очень низкая \\
\hline 3 & $\begin{array}{l}\text { Выполнение сочетающихя последовательных работ и работ, состоящих из } \\
\text { более мелких работ }\end{array}$ & Низкая \\
\hline 4 & Выполнение нерегламентированных определенным порядком работ & Нредняя \\
\hline 5 & Согласованное выполнение работ & Средняя \\
\hline 6 & Выполнение сочетающихся последовательных и согласуемых работ & Средняя \\
\hline 7 & $\begin{array}{l}\text { Поэтапное выполнение групп нерегламентированных определенным } \\
\text { порядком работ }\end{array}$ & Высокая \\
\hline 9 & Согласованное выполнение работ, состоящих из более мелких работ & Высокая \\
\hline 10 & $\begin{array}{l}\text { Выполнение нерегламентированных определенным порядком работ, } \\
\text { состоящих из более мелких работ } \\
\text { погласованное выполнение групп нерегламентированных определенным }\end{array}$ & Высокая \\
\hline
\end{tabular}

Сложный процесс содержит фрагменты, соответствующие типовым вариантам взаимодействия работ со средней или высокой сложностью, а простой - не содержит их, а включает лишь фрагменты, соответствующие типовым вариантам с очень низкой или низкой сложностью.

Так на основании анализа соответствия процессов типовым вариантам выявляются сложные и простые процессы, после чего выполняется переход ко второму этапу, если не требуется уточнение перечня процессов.

\section{Шаг 4. Уточнение перечня процессов}

Данный шаг является опциональным, и выполняется в случае, если при декомпозиции или в рамках 2-го этапа, который будет рассмотрен далее, выявляются новые сложные процессы.

Признаками необходимости уточнения является трудность:

- соотнесения процесса с одним из типовых вариантов проявления свойств (при выполнении шага 3); 
- учета существенных аспектов процесса и описания взаимодействия процессов (при выполнении шагов 5-7).

После уточнения перечня процессов осуществляется переход на 1-й шаг.

\section{Этап 2. Выполнение описания} коллективной деятельности

Описание коллективной деятельности на данном этапе выполняется, главным образом, нисходящим способом, начиная с построения процессной архитектуры и заканчивая простыми процессами.

В результате анализа методов, нотаций и примеров моделирования процессов [6-8] для выполнения описания коллективной деятельности выделено следующее множество элементов:

- основные объекты (работы, вызовы процесса, наборы работ, процесс);

- объекты для учета состояний (события потока управления, события приема-отправки сообщения);

- узлы (шлюзы ветвления и синхронизация);
- соединительные элементы (потоки управления, поток сообщений, связь с данными);

- данные;

- роли.

\section{Шаг 5. Построение процессной архитектуры}

При построении процессной архитектуры, абстрагированной от внутреннего содержания процессов, отражается информационное и событийное взаимодействие между процессами «одного уровня», а не прямая передача управления между ними.

На данном шаге применяются следующие элементы: процесс, поток сообщений, связь с данными, данные. Чаще всего с использованием этих элементов в процессной архитектуре осуществляется описание процессов, содержащих фрагменты средней и высокой сложности, соответствующих вариантам 5-10, как показано в табл. 4.

\section{Элементы, используемые для построения процессной архитектуры}

\begin{tabular}{|c|l|}
\hline № варианта & \multicolumn{1}{c|}{ Основные элементы описания } \\
\hline $1-4$ & Процесс \\
\hline $5-10$ & Процесс, поток сообщений, связь с данными, данные \\
\hline
\end{tabular}

При отсутствии фрагментов средней и высокой сложности, например, при проектном управлении, когда отдельные процессы не связаны и вызываются в виде реакции на какиелибо не типовые события, процессная архитектура вырождается и состоит из одного элемента для каждого процесса (варианты 1-4).

Обоснование построения процессной архитектуры приводится в статье [9]. Данный шаг является ключевым при выполнении описания коллективной деятельности, поскольку рассматриваемые сложные процессы относятся к различным субъектам деятельности (например, подразделениям), которые имеют собственные ритмы работы и поэтому их взаимодействие невозможно описать только с использованием потоков управления (в виде последовательности работ).

\section{Шаг 6. Описание сложных процессов}

На основании процессной архитектуры выполняется описание сложных процессов, выявленных на шаге 3. Для этого используется следующее подмножество элементов: работы, вызовы процесса, наборы работ, события потока управления и потоки управления, поток сообщений и прием-отправка сообщения, роли.

Исходя из соответствия сложного процесса (его фрагмента) некоторому варианту, при его описании целесообразно, главным образом, применять определенный для него набор элементов, как показано в табл. 5 (наименование вариантов приведено в табл. 3).

Элементы, используемые для описания сложных процессов.

\begin{tabular}{|c|l|}
\hline № варианта & \multicolumn{1}{|c|}{ Основные элементы описания } \\
\hline 1 & Работы, события потока управления, потоки управления \\
\hline 2 & Работы, вызовы процесса, события потока управления, потоки управления \\
\hline
\end{tabular}




\begin{tabular}{|c|l|}
\hline 3 & Работы, вызовы процесса, события потока управления, потоки управления \\
\hline 4 & Работы, наборы работ, события потока управления, потоки управления \\
\hline 5,6 & $\begin{array}{l}\text { Работы, события потока управления, потоки управления, поток сообщений, прием- } \\
\text { отправка сообщения, роли }\end{array}$ \\
\hline 7 & Работы, наборы работ, события потока управления, потоки управления, роли \\
\hline 8 & $\begin{array}{l}\text { Работы, вызовы процесса, события потока управления, потоки управления, поток } \\
\text { сообщений, прием-отправка сообения, роли }\end{array}$ \\
\hline 10 & $\begin{array}{l}\text { Работы, наборы работ, вызовы процесса, события потока управления, потоки управления, } \\
\text { роли }\end{array}$ \\
\hline Работы, наборы работ, события потока управления, потоки управления, поток сообщений, \\
прием-отправка сообщения, роли
\end{tabular}

При выявлении процессов, содержащих фрагменты очень низкой или низкой сложности, соответствующих вариантам $1-4$, их описание на данном шаге выполняется, если не существует возможности задать процесс без такого подробного представления этого фрагмента. В других случаях описание «простого» фрагмента проводится на следующем шаге.

\section{Шаг 7. Описание простых процессов}

Исходя из соответствия простого процесса (его фрагмента) некоторому варианту, содержащего фрагменты очень низкой или низкой сложности, при его описании используются наборы элементов, как показано в табл. 6.

\section{Элементы, используемые для описания простых процессов.}

Таблица 6

\begin{tabular}{|c|l|}
\hline № варианта & \multicolumn{1}{|c|}{ Основные элементы описания } \\
\hline 1 & $\begin{array}{l}\text { Процесс, работы, события потока управления, потоки управления, шлюзы, связь с данными, } \\
\text { данные, роли }\end{array}$ \\
\hline 2 & $\begin{array}{l}\text { Процесс, работы, вызовы процесса, события потока управления, потоки управления, шлюзы, } \\
\text { роли }\end{array}$ \\
\hline 3 & $\begin{array}{l}\text { Процесс, работы, вызовы процесса, события потока управления, потоки управления, шлюзы, } \\
\text { роли }\end{array}$ \\
\hline 4 & $\begin{array}{l}\text { Процесс, работы, наборы работ, события потока управления, потоки управления, шлюзы, } \\
\text { связь с данными, данные, роли }\end{array}$ \\
\hline 5 & $\begin{array}{l}\text { Процесс, работы, события потока управления, потоки управления, шлюзы, поток сообщений, } \\
\text { прием-отправка сообщения, роли }\end{array}$ \\
\hline
\end{tabular}

При выявлении на данном шаге фрагмента, соответствующего вариантам 5-10, требуется перейти к уточнению перечня процессов, в противном случае, после описания всех простых процессов, выполнение метода завершается.

В ходе проведения второго этапа предполагается применять способы формализованного описания и графического моделирования коллективной деятельности.

\section{II. Способ формализованного описания} коллективной деятельности

Предлагаемый способ формализованного описания коллективной деятельности заключается в составлении детерминированных и составных моделей деятельности на основе теории множеств с использованием моделей представления процессов, а также элементов алгебры поведений, алгебры взаимодействующих процессов и теории процессов [10-13].

\section{Пункт 1. Описание основных объектов, узлов и потоков управления}

Описание потока управления для отдельных работ $p_{k}, \quad p_{l}$ и $p_{m}$ в рамках процесса $P$ осуществляется согласно выражению, где операции над ними выполняются в указанном порядке:

$$
\begin{aligned}
& P=\left(p_{k}+p_{l}\right)-p_{m}=p_{k}+p_{l}-p_{m}, \\
& \text { а в общем виде }-P= \\
& p_{\text {[type.]name }}\left[+p_{\text {[type.]name }}\right]\left[-p_{[\text {type.]name }}\right] \text {, } \\
& \text { где }=-\quad \text { вызов содержимого процесса; } \\
& + \text { - операция одновременного } \\
& \text { выполнения независимых работ; } \\
& \text { - - операция последовательного }
\end{aligned}
$$




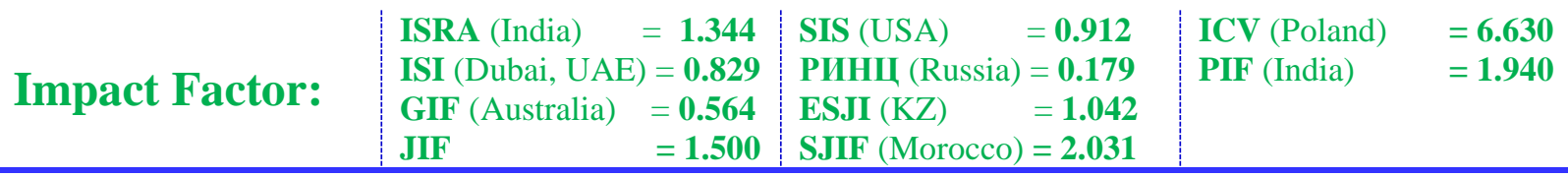

выполнения работ;

$p_{\text {[type.]name }}$ указание типа и (или) наименования работы (процесса) $p$

[] - указание необязательного элемента.

Описание потока управления с условиями, обеспечивающими выбор альтернативных работ в процессе, осуществляется следующим образом:

$$
P=p_{k}: \text { condition }_{\text {default }} \div p_{l}: \text { rule }_{r},
$$

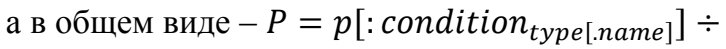
$p\left[\right.$ : condition $\left._{\text {type }[\text {.name }]}\right]$,

где $\div-$ операция выбора для выполнения одной из нескольких работ;

condition $_{\text {type[name] }}$ условие выбора работы для выполнения с указанием типа условия и при необходимости (для пользовательского правила) наименования.

При этом условие выбора работы может отсутствовать, иметь флаг «по умолчанию», либо быть описанным в виде некоторого правила из множества бизнес-правил:

$$
\begin{gathered}
\text { type }(\text { condition }\langle\div\rangle)=\left\{\varnothing, \text { default },\left\{\text { rule }_{\text {name }}\right\}\right\}, \\
\text { rule }_{\text {name }} \in \text { Rule. }
\end{gathered}
$$

Для описания набора работ, выполнение которых не регламентировано определенным порядком, т. е. где поток управления не может быть жестко задан, введена операция неупорядоченного выполнения работ:

$$
\begin{aligned}
& P=p_{k} \sim p_{l} \sim p_{m}: \text { condition }_{\text {one_task }_{\text {ta }},}, \\
& \text { а в общем виде }-P= \\
& p \sim p\left[\text { condition }_{\text {type }[\text {.name }]}\right], \\
& \text { где - операция } \\
& \text { неупорядоченного } \\
& \text { выполнения нескольких } \\
& \text { работ; }
\end{aligned}
$$

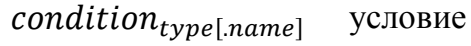

$$
\begin{aligned}
& \text { - неупорядоченного } \\
& \text { выполнения работ. }
\end{aligned}
$$

Условие неупорядоченного выполнения может отсутствовать, быть типов «транзакция», «выполнить единственную работу», «выполнить все работы», либо быть описанным в виде некоторого правила:

$$
\begin{aligned}
& \text { type }(\text { condition }\langle\sim\rangle)=
\end{aligned}
$$

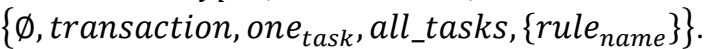

Транзакция при этом обеспечивает выполнение либо всех работ, либо невыполнение ни одной (откат результатов выполненных работ) - transaction $=\emptyset$ vall_tasks .
В качестве работ в приведенных выражениях могут выступать работы следующих основных типов:

$$
\begin{aligned}
\text { type }(\text { task }) & =\{\text { user, service, script, abstract }\}, \\
\text { где user }- & \text { тип пользовательского задания с } \\
& \text { ручным выполнением; } \\
\text { service }- & \text { тип автоматизированного } \\
& \text { пользовательского задания; } \\
\text { script }- & \text { тип автоматического задания; } \\
\text { abstract } & \text { абстрактный тип задания (без } \\
- & \text { указания типа) }
\end{aligned}
$$

или независимые процессы $p_{k}, p_{l}, p_{m} \in P$, которые вместе с предложенными операциями над работами:

$$
\begin{aligned}
& \text { + - параллельной композиции работ; } \\
& \text { - - последовательной композиции работ; } \\
& \text { - - альтернативной композиции работ; } \\
& \text { - неупорядоченной композиции работ, }
\end{aligned}
$$

покрывают такие элементы для выполнения описания деятельности как - потоки управления, шлюзы ветвления и синхронизации, а также работы, вызовы процесса, наборы работ и процесс.

\section{Пункт 2. Описание объектов для учета состояний}

При дальнейшей детализации процессов используются элементы, описывающие события потока управления, которые определяют начало и конец работы, отмену и ошибку выполнения и т. д. Для их задания применяются следующие основные типы событий:

$$
\begin{gathered}
\text { type }(\text { event })= \\
\{\text { plain, cancel, error, timer, signal }\},
\end{gathered}
$$

где plain тип простого события (начала или - окончания процесса);

cancel - тип события, инициирующего отмену (реагирующего на отмену) работы;

error - тип события, инициирующего генерацию (обработку) ошибки при выполнении работы;

timer - тип события, регулярно происходящего во времени;

signal - тип события, рассылающего (принимающего) сигналы между несколькими процессами.

Описание использования сигналов в процессе выполняется следующим образом:

$$
\begin{aligned}
& P=p\left[\cdot s_{\text {type }[\text {.subtype }][\text {.name }]}\right], \\
& \text { где } \cdot-\text { операция добавления } \\
& \text { события относительно } \\
& \text { некоторой работы; }
\end{aligned}
$$




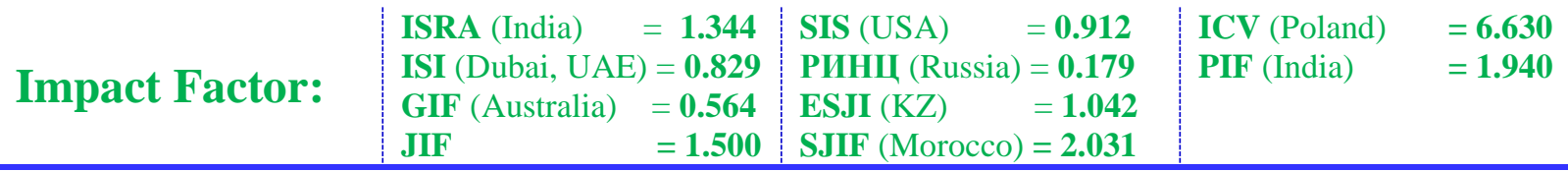

$S_{\text {type[.subtype][.name] }}-$ указание типа, а также подтипа и наименования события при необходимости.

С учетом того, что многие из приведенных типов событий могут быть начальными, промежуточными или завершающими процесс событиями, продемонстрируем несколько вариантов описания фрагментов, их содержащих. Так, фрагмент процесса, описывающий:

- простое начало процесса, имеет вид $-\delta$. $S_{\text {plain.start, }}$ где $\delta$ - пустая работа, start обозначение подтипа «начало процесса» простого события;

- срабатывание таймера во время выполнения автоматизированного пользовательского задания «проверка данных», задается в виде $-p_{\text {user.check }} \cdot s_{\text {timer }}$;

- обработку ошибки «недостаточно памяти» при выполнении процесса «тестирование», имеет

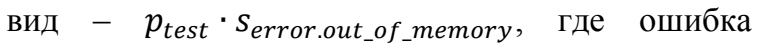
такого типа может возникать как внутри $p_{\text {test }}$, так и при выполнении другого, параллельного процесса;

- завершение некоторого процесса с ошибкой «недостаточно памяти», задается в виде $-\delta \cdot s_{\text {error.out_of_memory }}$

- простое завершение процесса, имеет вид $\delta \cdot s_{\text {plain.end }}$, где end - обозначение подтипа «окончание процесса» простого события;

- отправку сигнала «тревога», задается в

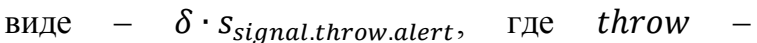
обозначение подтипа «рассылка» сигнального события;

- обработку сигнала «тревога», ведущего к прерыванию процесса, к которому он добавлен, имеет вид $-p_{\text {sleep }} \cdot s_{\text {signal.catch.alert }}$, где catch обозначение подтипа «ожидание и прием» сигнала.

Аналогичным образом выполняется описание событий, связанных с приемомотправкой сообщений с данными, для которых дополнительно указывается входной или выходной объект данных из множества внутренних данных процессов:

$P=\left[\cdot d_{\text {in }} ? s_{\text {mes.catch.name }}\right] p\left[\cdot s_{\text {mes.throw.name }} ! d_{\text {out }}\right]$,

где ? (!) - операция префиксинга, т. е. добавления к элементу, ожиданияприема (вывода) объекта данных относительно сообщения.

Приведенные выражения позволяют описывать объекты для учета состояний и события приема-отправки сообщений.

Пункт 3. Описание данных, потока сообщений и связи с данными
Данные, используемые при описании деятельности, могут относиться к внутренним или внешним объектам данных - Data = Data $_{\text {int }} \cup$ Data $_{\text {ext }}$. Внутренними являются данные самих процессов, которые применяются при их выполнении:

$$
\text { Data }_{\text {int }}=\left(d_{\text {in }}, d_{\text {out }}\right) \text {, }
$$

а в общем виде - Data $_{\text {int }}=\left\{d_{\text {name }}\right\}$,

где $d_{\text {naте }}$ именованный объект внутренних - данных.

К внешним данным относятся хранилища данных типа база данных, или отдельно обозначенных, логически выделенных объектов данных, например, «заказы» и «расписание производства»:

$$
\text { Data }_{\text {ext }}=\left(B_{\text {orders }}, B_{\text {schedule }}\right),
$$

а в общем виде - Data $_{\text {ext }}=\left\{B_{\text {name }}\right\}$,

где $B_{\text {name }}$ именованный объект внешних - данных.

Передача внутренних данных в рамках процесса обозначается подобно рассмотренному выше выражению с приемом-отправкой сообщений:

$$
P=\left[d_{\text {in }} ?\right] p\left[! d_{\text {out }}\right], d_{\text {in }}, d_{\text {out }} \in \text { Data }_{\text {int }},
$$

где ? (!) - операция приема (вывода) объекта данных относительно работы.

Подобное выражение используется и для описания связи с внешними источниками данных, например для получения «заказов» при выполнении пользовательской работы «Планирование», в результате чего формируется объект данных «расписание производства» (в данном выражении - с указанием сигналов начала и конца процесса):

$$
\begin{gathered}
P=\delta \cdot s_{\text {plain.start }}- \\
-B_{\text {orders }} ? p_{\text {user.planning }} ! B_{\text {schedule }}- \\
-\delta \cdot s_{\text {signal.throw.ready }} .
\end{gathered}
$$

Приведенные выражения позволяют описывать поток сообщений, связь с данными, а также указывать сами данные при формализации коллективной деятельности.

\section{Пункт 4. Описание ролей}

Для отражения ролей используется следующее выражение, которое позволяет задавать различные варианты для назначения исполнителей работ (например, назначение «управляющего» или «директора» для выполнения работы «планирование» в указанном порядке):

$$
\begin{aligned}
& \text { Roles }=\left(p_{\text {user.planning }} \rightarrow\right. \\
& \left.\left(R_{\text {manager }}, R_{\text {director }}\right): \text { condition } \text { successively }\right) \text {, } \\
& \text { а в общем виде }- \text { Roles }=\left\{p_{\text {type }[\text {.name }]} \rightarrow\right. \\
& \left.\left\{R_{\text {name }}\right\}\left[\text { : condition }_{\text {type }[\text {.name }]}\right]\right\} \text {, }
\end{aligned}
$$




\begin{tabular}{|c|c|c|c|c|c|c|}
\hline Impact Factor: & $\begin{array}{l}\text { ISRA (India) } \\
\text { ISI (Dubai, UAE } \\
\text { GIF (Australia) } \\
\text { JIF }\end{array}$ & $\begin{array}{l}=1.344 \\
=0.829 \\
=0.564 \\
=1.500\end{array}$ & $\begin{array}{l}\text { SIS (USA) = } \\
\text { PИНЦ (Russia) } \\
\text { ESJI (KZ) } \\
\text { SJIF (Morocco) }\end{array}$ & $\begin{array}{l}=0.912 \\
=0.179 \\
=1.042 \\
=2.031\end{array}$ & $\begin{array}{l}\text { ICV (Poland) } \\
\text { PIF (India) }\end{array}$ & $\begin{array}{l}=6.630 \\
=1.940\end{array}$ \\
\hline
\end{tabular}

$$
\begin{aligned}
\text { где } \rightarrow- & \text { отношение соответствия } \\
& \text { ролей и работ; } \\
\text { condition }_{\text {type[name }]} & \text { условие выбора ролей } \\
- & \text { для выполнения работы. }
\end{aligned}
$$

Особенностью этой записи является то, что для одной и той же работы могут быть определены несколько ролей-кандидатов, каждая из которых (a по сути, сотрудник еe исполняющий) сможет взять на выполнение конкретный экземпляр задания, пока это не сделали другие кандидаты согласно условию выбора ролей.

Условие выбора ролей может отсутствовать, иметь флаг «по порядку», либо описываться в виде некоторого бизнес-правила:

$$
\begin{gathered}
\text { type }(\text { condition }\langle\rightarrow\rangle)= \\
\left\{\varnothing, \text { successively, }\left\{\text { rule }_{\text {name }}\right\}\right\} .
\end{gathered}
$$

Таким образом, указанные в пунктах 1-4 выражения позволяют использовать при формализации все элементы, необходимые для описания коллективной деятельности, а предложенный способ - составлять модели деятельности, обеспечивающие:

- представление коллективной деятельности различного характера и согласованного взаимодействия процессов;

- учет данных внутри процессов и связей с внешними источниками данных, а также бизнесправил, ролей и других условий выполнения работ.

\section{III. Способ графического моделирования коллективной деятельности}

Целью этого способа не является создание нового графического языка моделирования, поскольку для документирования и моделирования автоматизированной деятельности, бизнес-процессов, потоков или структуры работ существует множество графических нотаций и вариантов их реализации [6-8]. Также при описании способа не рассматриваются вопросы анализа данных по результатам многократного проигрывания процессов (так называемая симуляция и аналитика бизнес-процессов).

Предлагаемый способ графического моделирования направлен на обобщение лучших техник и приемов, проверки шаблонов применения различных ВРМ-систем, а также введения новых паттернов для описания коллективной деятельности.

Для этого на примере стандарта BPMN приведем соответствие элементов описания коллективной деятельности и графических элементов этой нотации (табл. 7). Следует

\begin{tabular}{|c|c|c|}
\hline $\begin{array}{l}\text { Группы элементов } \\
\text { описания }\end{array}$ & $\begin{array}{l}\text { Основные элементы } \\
\text { описания }\end{array}$ & Элементы нотации BPMN \\
\hline \multirow[t]{4}{*}{ Основные объекты } & Работы & Задание (задача) \\
\hline & Вызовы процесса & Свернутый подпроцесс, подпроцесс-вызов \\
\hline & Наборы работ & Ad-hoc-подпроцесс \\
\hline & Процесс & Пул, свернутый пул, развернутый подпроцесс \\
\hline \multirow[t]{2}{*}{$\begin{array}{l}\text { Объекты для учета } \\
\text { состояний }\end{array}$} & $\begin{array}{ll}\text { События } & \text { потока } \\
\text { управления } & \\
\end{array}$ & $\begin{array}{l}\text { Простые события, события-отмены, события-ошибки, } \\
\text { события-таймеры, события-сигналы и т. д. }\end{array}$ \\
\hline & $\begin{array}{l}\text { События приема-отправки } \\
\text { сообщения }\end{array}$ & События-сообщения \\
\hline Узлы & $\begin{array}{l}\text { Шлюзы ветвления } \quad \text { и } \\
\text { синхронизация }\end{array}$ & $\begin{array}{l}\text { Шлюзы: параллельный шлюз, эксклюзивный шлюз, } \\
\text { комплексный шлюз, эксклюзивный шлюз по } \\
\text { событиям и т. д. }\end{array}$ \\
\hline \multirow[t]{3}{*}{$\begin{array}{l}\text { Соединительные } \\
\text { элементы }\end{array}$} & Потоки управления & $\begin{array}{l}\text { Поток управления, условный поток, поток по } \\
\text { умолчанию }\end{array}$ \\
\hline & Поток сообщений & Поток сообщений \\
\hline & Связь с данными & Ассоциации \\
\hline Данные & Данные & Объект данных, база данных \\
\hline Роли & Роли & Дорожки \\
\hline
\end{tabular}
отметить, что производители ВРМ-систем зачастую реализуют лишь некоторую часть элементов данной нотации.

\section{Соответствие элементов описания коллективной деятельности и нотации ВРМN}




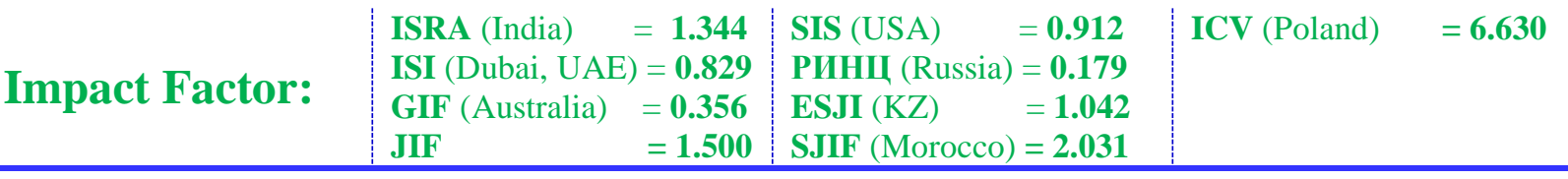

Приведенное соответствие позволяет обосновать наличие того или иного элемента в конкретной нотации или выявить отсутствие, например, набора работ (Ad-hoc-подпроцесса) в реализации стандарта ВPMN 2.0 в системе Activiti [8].

Определив используемые для графического моделирования элементы, перейдем к рассмотрению примеров коллективной деятельности, соответствующих типовым вариантам взаимодействия работ со средней или высокой сложностью. В ходе этого предполагается обосновать некоторые приемы описания коллективной деятельности. Подчеркнем, что при этом будут приведены диаграммы не реальных процессов, а фрагментов, отражающих особенности в части сочетания отдельных свойств коллективной деятельности.

\section{Прием 1. Описание последовательного выполнения согласуемых работ}

Описание последовательного выполнения работ, согласуемых в рамках единого процесса, целесообразно проводить не в одном пуле, а в виде нескольких процессов, взаимодействующих посредством сигналов, сообщений или данных. Это обосновывается тем, что при производстве такие взаимодействующие процессы соответствуют циклам работ, связанным с изготовлением продукта различными подразделениями предприятия [14].

Для демонстрации рассмотрим фрагмент выполнения работ от приема заказа до производства. В соответствии с приведенной рекомендацией в данном случае выделено три процесса - прием заказа, планирование и производство, как показано на рис. 11.
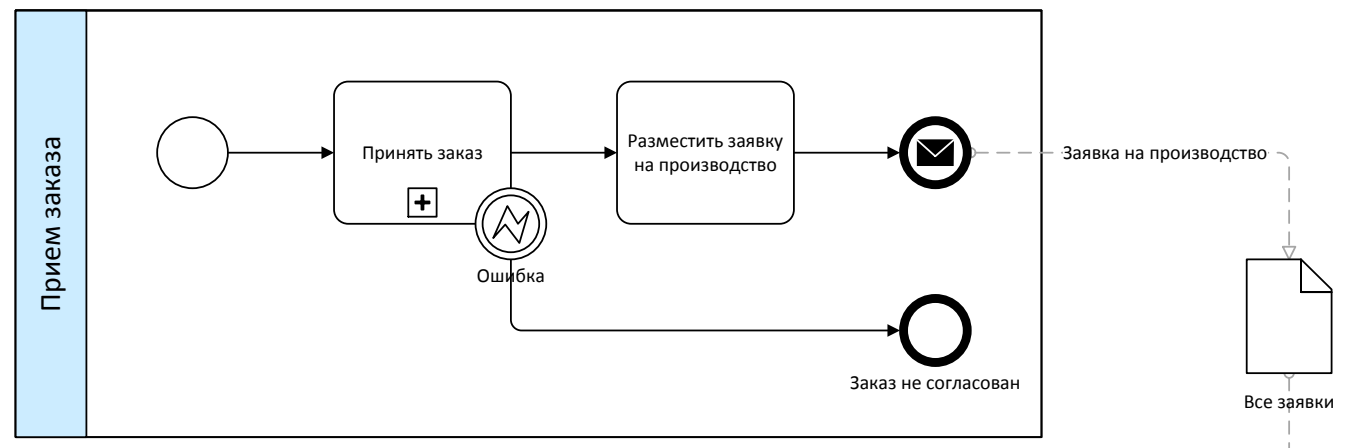

Все заявки
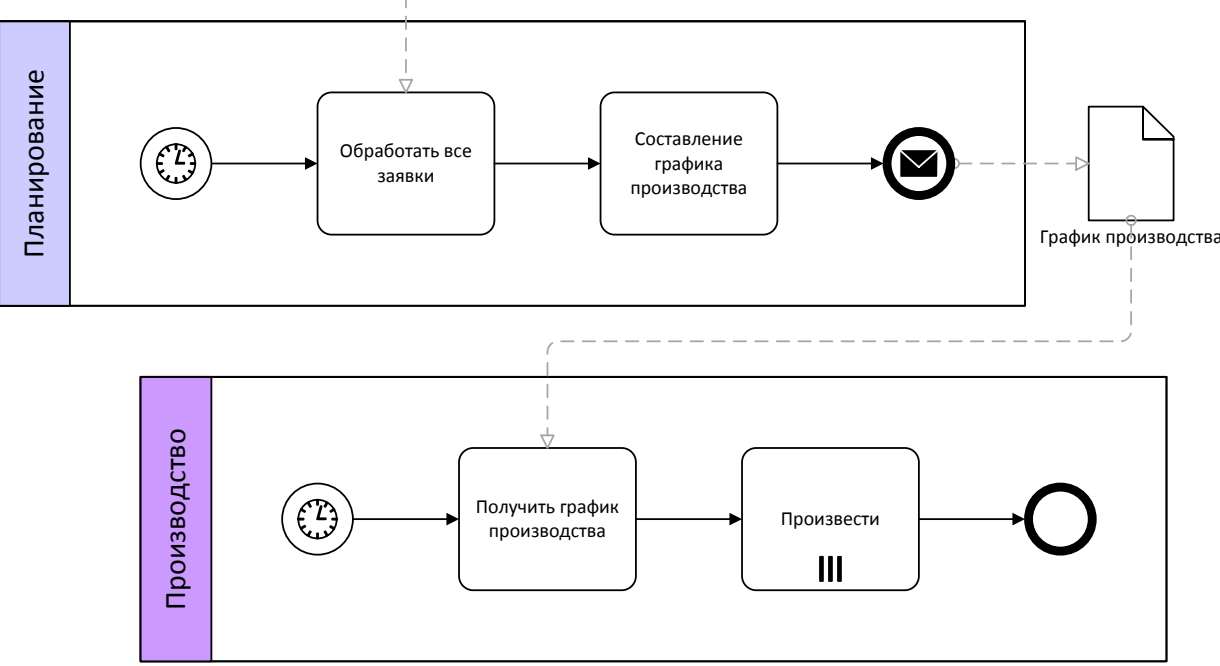

Рисунок 11 - Выполнение работ от приема заказа до производства

Такое разбиение на процессы связано с тем, что «производство не подчиняется ритму поступления клиентских заказов, т. к. ему необходимо заблаговременно спланировать настройку оборудования, обеспечение материалами и полуфабрикатами, поэтому процесс выполнения клиентского заказа не дирижирует производством» [15]. При этом для обработки всех заявок и составления графика производства для распределения имеющихся ресурсов при выполнении заказов вводится процесс планирования, который запускается периодически, как процесс производства. 


\begin{tabular}{|c|c|c|c|c|c|c|}
\hline Impact Factor: & $\begin{array}{l}\text { ISRA (India) } \\
\text { ISI (Dubai, UAF } \\
\text { GIF (Australia) } \\
\text { JIF }\end{array}$ & $\begin{array}{l}=1.344 \\
=0.829 \\
=0.564 \\
=1.500\end{array}$ & $\begin{array}{l}\text { SIS (USA) } \\
\text { PИНЦ (Russia) } \\
\text { ESJI (KZ) } \\
\text { SJIF (Morocco) }\end{array}$ & $\begin{array}{l}=0.912 \\
=0.179 \\
=1.042 \\
=2.031\end{array}$ & $\begin{array}{l}\text { ICV (Poland) } \\
\text { PIF (India) }\end{array}$ & $\begin{array}{l}=6.630 \\
=1.940\end{array}$ \\
\hline
\end{tabular}

Прием 2. Описание регламентированного взаимодействия независимых субъектов

При описании регламентированного взаимодействия независимых субъектов, при котором сочетаются свойства последовательности и согласованности деятельности, необходимо учитывать различные варианты ответной реакции на поведение субъекта из внешнего мира.

Для примера рассмотрим фрагмент взаимодействия с клиентом при выставлении ему счета и ожидании получения оплаты от него. В данном случае, как показано на рис. 12, выделено два процесса, поскольку в рамках этого фрагмента рассматривается взаимодействие двух независимых субъектов, один из которых не раскрыт, поскольку его поведение не регламентируется, а во втором процессе реализовано:

- реагирование на все возможные действия клиента, т. е. «обработка альтернативных сообщений, когда необходимо ожидать одновременно всех событий от независимого субъекта, а не только планируемого» [16];

- фиксирование исходов по результатам взаимодействия.

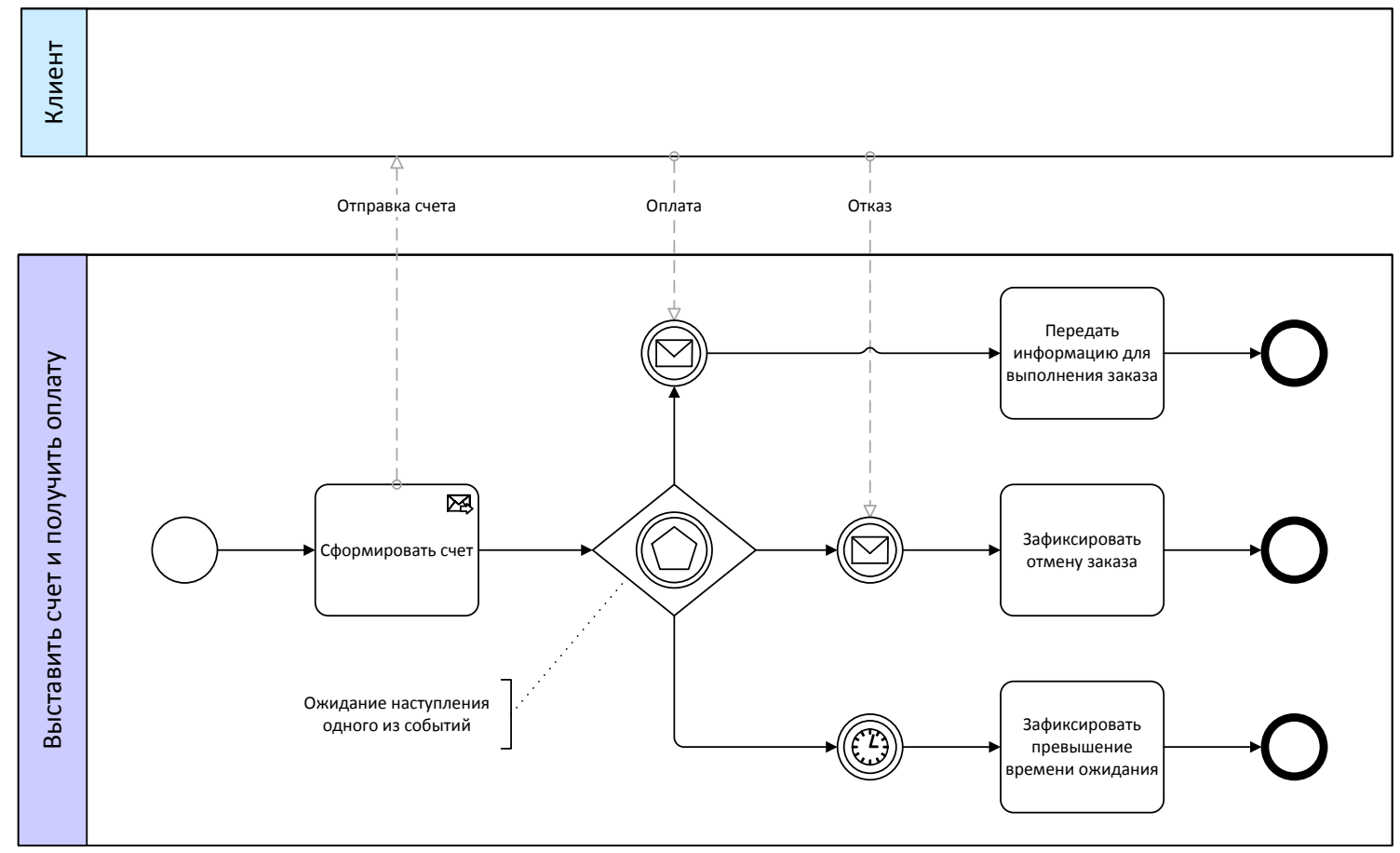

Рисунок 12 - Взаимодействие с клиентом

Прием 3. Описание регламентированного взаимодействия зависимых субъектов

При описании регламентированного взаимодействия зависимых субъектов, при котором сочетаются свойства согласованности и вложенности деятельности, необходимо управлять выполнением всех работ нижестоящего уровня.
Для демонстрации рассмотрим фрагмент деятельности при подготовке, оценке и рассмотрении решения на взаимоподчиненных уровнях. В данном случае, как показано на рис. 13, выделено три процесса, обеспечивающих взаимодействие трех субъектов, начинающих выполнение работ «по команде сверху» или по готовности документов от подчиненных. 


\begin{tabular}{l|lrl|l|ll} 
& ISRA (India) & $=\mathbf{1 . 3 4 4}$ & SIS (USA) & $=\mathbf{0 . 9 1 2}$ & ICV (Poland) & $=\mathbf{6 . 6 3 0}$ \\
Impact Factor: & ISI (Dubai, UAE) $=\mathbf{0 . 8 2 9}$ & PUHU (Russia) $=\mathbf{0 . 1 7 9}$ & PIF (India) & $=\mathbf{1 . 9 4 0}$ \\
& GIF (Australia) & $\mathbf{0 . 5 6 4}$ & ESJI (KZ) & $=\mathbf{1 . 0 4 2}$ & & \\
& JIF & $=1.500$ & SJIF (Morocco) & $=\mathbf{2 . 0 3 1}$ & &
\end{tabular}
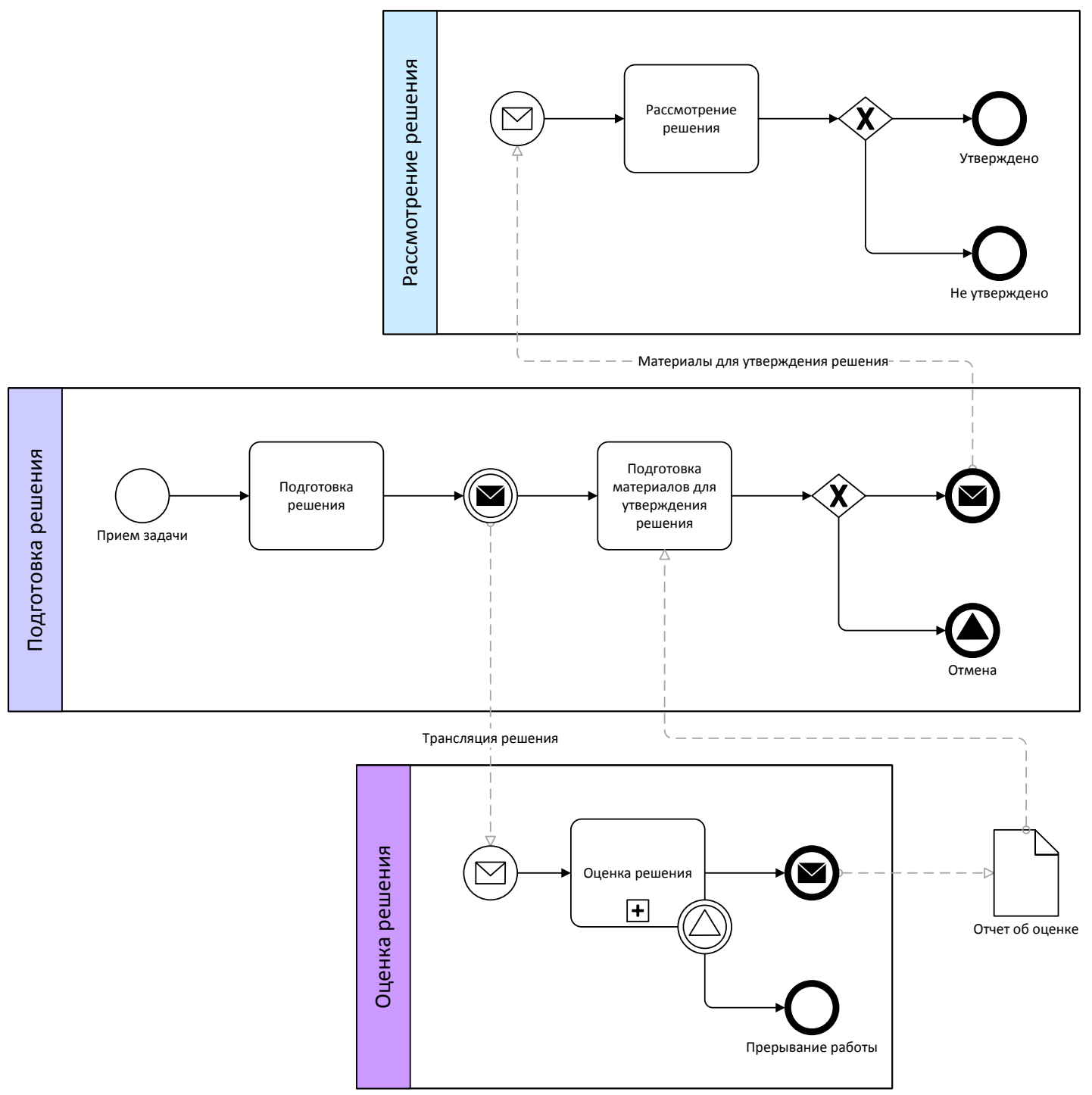

Рисунок 13 - Взаимодействие при подготовке, оценке и рассмотрении решения

Один из подчиненных может по своему усмотрению прервать работу подчиненного самого нижнего уровня, либо, не дожидаясь его готовности, отправить подготовленные самим материалы на рассмотрение вышестоящему начальнику, который принимает решение.

Для обеспечения управления выполнением работ на нижестоящем уровне, здесь реализовано взаимодействие через элементы:

- сообщения, определяющие начало работы «по команде сверху»;

- сигналы, прерывающие работу соседнего процесса любой момент;

- данные, позволяющие «переносить» контроль управления работой из схемы на уровень пользователя, когда достаточность полученных данных или не критичность их неполучения определяет участник деятельности, а не формализованный регламент.
Прием 4. Описание взаимодействия зависимых субъектов с нерегламентированным поведением

При описании взаимодействия зависимых субъектов с нерегламентированным поведением, при котором сочетаются свойства индивидуальности и вложенности деятельности, необходимо обеспечивать синхронизованное и непрерывное выполнение всех работ на нижестоящем уровне.

Для примера рассмотрим фрагмент взаимодействия при постановке задачи подчиненному и ее выполнении, как показано на рис. 14. Подчиненный при этом выполняет нерегламентированные определенным порядком работы, а также без их прерывания (потоком управления) реагирует на необходимость «внести изменения» по команде вышестоящего, когда уже приступил к решению поставленной ему задачи. 


\begin{tabular}{|c|c|c|c|c|c|c|}
\hline Impact Factor: & $\begin{array}{l}\text { ISRA (India) } \\
\text { ISI (Dubai, UAE } \\
\text { GIF (Australia) } \\
\text { JIF }\end{array}$ & $\begin{array}{l}=1.344 \\
=0.829 \\
=0.356 \\
=1.500\end{array}$ & $\begin{array}{l}\text { SIS (USA) } \\
\text { PИHЦ (Russia) } \\
\text { ESJI (KZ) } \\
\text { SJIF (Morocco) }\end{array}$ & $\begin{array}{l}=0.912 \\
=0.179 \\
=1.042 \\
=2.031\end{array}$ & ICV (Poland) & $=6.630$ \\
\hline
\end{tabular}
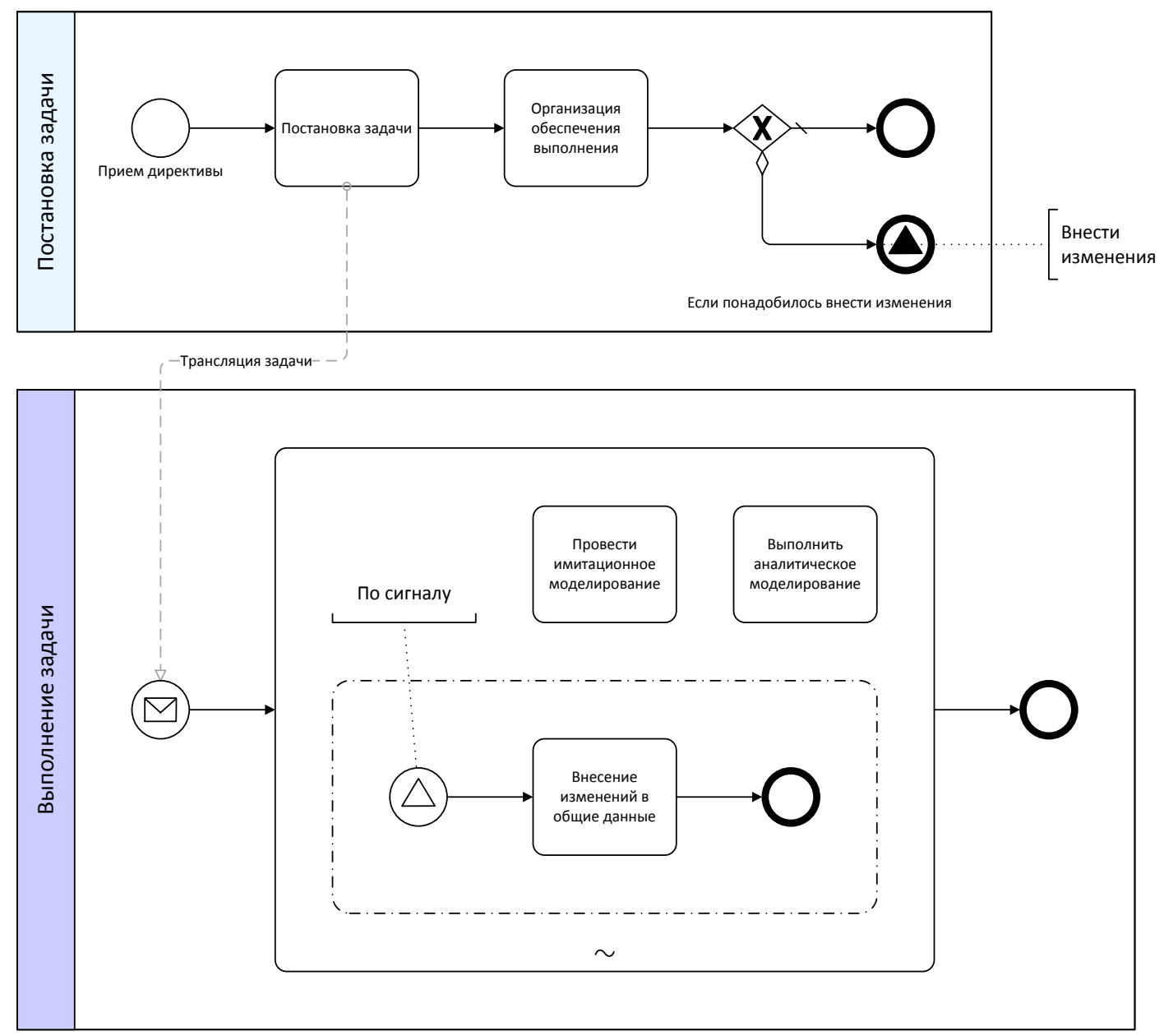

Рисунок 14 - Взаимодействие при постановке и выполнении задачи

Приведенная диаграмма позволяет отражать работы подчиненного, которые могут начинаться и завершаться независимо друг от друга (элемент «набор работ»), как им самим, так и «по команде сверху» без прерывания других работ. Различные варианты использования элемента «событийный вызов процесса» описаны в [17]. Особенностью применения этих элементов является возможность обработки общих данных при выполнении нерегламентированных определенным порядком работ.

Прием 5. Описание выполнения нерегламентированных определенным порядком работ
При описании выполнения нерегламентированных работ, при котором сочетаются свойства последовательности и индивидуальности, в определенных случаях не целесообразно жестко задавать порядок, а обеспечивать согласование работ через данные.

Рассмотрим фрагмент взаимодействия при оценке решения, в котором, как показано на рис. 15, выделены обязательные для выполнения работы и необязательные работы. 


\begin{tabular}{l|lrl|l|ll} 
& ISRA (India) & $=\mathbf{1 . 3 4 4}$ & SIS (USA) & $=\mathbf{0 . 9 1 2}$ & ICV (Poland) & $=\mathbf{6 . 6 3 0}$ \\
Impact Factor: & ISI (Dubai, UAE) $=\mathbf{0 . 8 2 9}$ & PUHU (Russia) $=\mathbf{0 . 1 7 9}$ & PIF (India) & $=\mathbf{1 . 9 4 0}$ \\
& GIF (Australia) & $\mathbf{0 . 5 6 4}$ & ESJI (KZ) & $=\mathbf{1 . 0 4 2}$ & & \\
& JIF & $=1.500$ & SJIF (Morocco) & $=\mathbf{2 . 0 3 1}$ & &
\end{tabular}

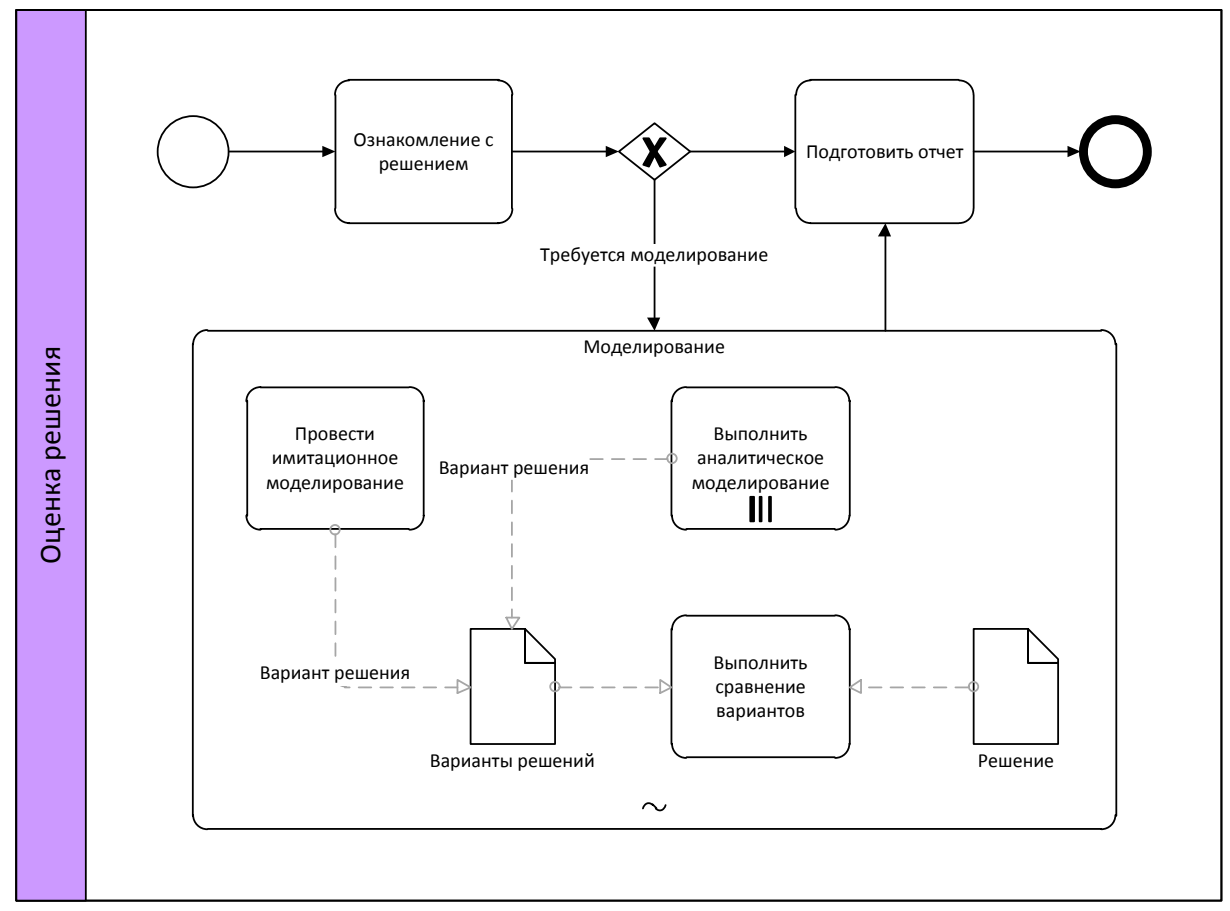

Рисунок 15 - Взаимодействие при оценке решения

При этом порядок выполнения обязательных работ задан напрямую (ознакомление с решением, подготовка отчета), а связанных с моделированием необязательных работ - не регламентирован. Работы по моделированию, проведению расчетов и сравнению результатов могут осуществляться в любом порядке и согласуются через данные, т. е. не связаны потоками управления.

В целом, обобщение удачных примеров и выделение на их основе общих приемов описания коллективной деятельности позволяет упростить процесс моделирования и избежать допущения типовых ошибок за счет повторного использования апробированных приемов. Следует учитывать, что список приведенных приемов не претендует на полноту, должен расширяться и детализироваться для конкретных предметных областей (с целью учета существенных особенностей конкретной области).

\section{Заключение}

Предложенный метод согласованного представления коллективной деятельности включает систематизированный порядок проведения действий, разбитый на этапы анализа и выполнения описания, и применения способов формализованного описания и графического моделирования коллективной деятельности.

Особенностью метода является то, что в нем предлагается:
- выявлять процессы, относящиеся (не относящиеся) к коллективной деятельности, согласно установленным признакам процессов;

- определять факт общности процессов в рамках всей деятельности;

- выявлять в процессах фрагменты, позволяющие отнести их к категории простых или сложных процессов;

- выполнять описание деятельности на основании набора элементов, заданного для каждого из уровней представления (архитектура, сложные и простые процессы), с использованием предложенных способов формализованного описания и графического моделирования коллективной деятельности.

Эти способы в рамках метода обеспечивают соответствующие для математического и графического представления коллективной деятельности уровни адекватности и подробности за счет учета:

- существенных особенностей коллективной деятельности, связанных с разнохарактерностью и субъективностью выполнения отдельных процессов и согласования деятельности в целом;

- связей с различными источниками данных;

- бизнес-правил, ролей и других условий выполнения работ.

Таким образом, предложенный метод согласованного представления коллективной деятельности позволяет проводить формализованное описание и графическое моделирование деятельности, выявлять неявные участки, верифицировать и устанавливать порядок выполнения коллективной деятельности. 
Исследование выполнено при финансовой поддержке РФФИ в рамках научного проекта № 14-07-00338_а, а также в рамках базовой части государственного задания Минобрнауки России
№ 2014/123 на выполнение государственных работ в сфере научной деятельности, проект № 2493 .

\section{References:}

1. Zabezhajlo $\mathrm{M}$ (2010) $\mathrm{K}$ voprosu o vybore adekvatnoj metodologii transformacii biznesa krupnogo kommercheskogo banka. Vestnik RGGU. Serija: Dokumentovedenie i arhivovedenie. Informatika. Zashhita informacii i informacionnaja bezopasnost', no. 12, pp. 4663.

2. Belaychuk A (2014) Chto javljaetsja processom v BPMN (i chto ne javljaetsja), Available: http://mainthing.ru/ru/item/715/ (Accessed: 18.09.2015).

3. Mansurov A (2013) Upravlenie chastnogosudarstvennym partnerstvom kak edinstvo processnogo i proektnogo menedzhmenta. Biznes. Obrazovanie. Pravo. Vestnik volgogradskogo instituta biznesa, no. 4, pp. 303-306.

4. Rudkovskij I (2013) Reshenie zadach logisticheskogo menedzhmenta na osnove proektno-orientirovannogo upravlenija. Izvestija Sankt-Peterburgskogo gosudarstvennogo jekonomicheskogo universiteta, no. 5, pp. 92-96.

5. Syskov V, Borisov V (2015) Podhod k postroeniju sistemy intellektual'nogo processnogo upravlenija dlja obespechenija jeffektivnoj kollektivnoj dejatel'nosti. Upravlenie jekonomicheskimi sistemami: jelektronnyj nauchnyj zhurnal, no. 10, Available:

http://uecs.ru/index.php?option=com_flexiconte nt\&view=items\&id=3785

(Accessed: 18.09.2015)

6. Business Process Model and Notation (BPMN). Version $2.0 \quad$ (2011), Available: http://www.omg.org/spec/BPMN/2.0

(Accessed: 18.09.2015).

7. Mayer R, Menzel C, Painter M (1995) Information Integration for Concurrent Engineering (IICE). IDEF3 Process Description Capture Method Report. College Station, TX: Knowledge Based Systems, Inc., 224 p.

8. Activiti User Guide. Version 5.18.0 (2015), Available: http://activiti.org/userguide/index.html (Accessed: 18.09.2015).

9. Belaychuk A (2014) Mezhprocessnoe vzaimodejstvie cherez dannye, Available: http://mainthing.ru/ru/item/332/ (Accessed: 18.09.2015).

10. Syskov V, Borisov V (2014) Postroenie modelej predstavlenija dejatel'nosti dlja realizacii processnogo upravlenija $\mathrm{v}$ slozhnyh organizacionno-tehnicheskih sistemah. Matematicheskaja morfologija. Jelektronnyj matematicheskij i mediko-biologicheskij zhurnal, vol. 13, no. 4, Available: http://www.smolensk.ru/user/sgma/MMORPH/ N-44-html/borisov/borisov.htm (Accessed: 18.09.2015).

11. Bergstra J, Klop J (1984) Process algebra for synchronous communication. Information and Control, no. 60, pp. 109-137.

12. Milner R (1986) Calculus of Communicating Systems. Edinburgh: University of Edinburgh, $171 \mathrm{p}$.

13. Mironov A (2008) Teorija processov. Pereslavl'-Zalesskij: Universitet goroda Pereslavlja, $344 \mathrm{p}$.

14. Benedict T, Bilodeau N, Vitkus P (2013) BPM CBOK Version 3.0: Guide to the Business Process Management Common Body Of Knowledge. ABPMP, 443 p.

15. Belaychuk A (2013) Dirizhirovat' ili reagirovat', Available: $\quad$ http://mainthing.ru/ru/item/613/ (Accessed: 18.09.2015).

16. Belaychuk A (2010) Processnyj antipattern: garantirovannoe poluchenie soobshhenija, Available: $\quad$ http://mainthing.ru/ru/item/273/ (Accessed: 18.09.2015).).

17. BPMN 2.0 new features - Event Sub-Process (2010), Available: http://www.quweiji.com/bpmn-2-0-newfeatures-event-sub-process-event-sub-process/ (Accessed: 18.09.2015). 